\title{
Rattle: A Data Mining GUI for R
}

\section{by Graham J Williams}

\begin{abstract}
Data mining delivers insights, patterns, and descriptive and predictive models from the large amounts of data available today in many organisations. The data miner draws heavily on methodologies, techniques and algorithms from statistics, machine learning, and computer science. $\mathrm{R}$ increasingly provides a powerful platform for data mining. However, scripting and programming is sometimes a challenge for data analysts moving into data mining. The Rattle package provides a graphical user interface specifically for data mining using $R$. It also provides a stepping stone toward using $R$ as a programming language for data analysis.
\end{abstract}

\section{Introduction}

Data mining combines concepts, tools, and algorithms from machine learning and statistics for the analysis of very large datasets, so as to gain insights, understanding, and actionable knowledge.

Closed source data mining products have facilitated the uptake of data mining in many organisations. These products offer off-the-shelf ease-of-use that makes them attractive to the many new data miners in a market place desperately seeking high levels of analytical skills.

$\mathrm{R}$ is ideally suited to the many challenging tasks associated with data mining. $\mathrm{R}$ offers a breadth and depth in statistical computing beyond what is available in commercial closed source products. Yet R remains, primarily, a programming language for the highly skilled statistician, and out of the reach of many.

Rattle (the R Analytical Tool To Learn Easily) is a graphical data mining application written in and providing a pathway into R (Williams, 2009b). It has been developed specifically to ease the transition from basic data mining, as necessarily offered by GUIs, to sophisticated data analyses using a powerful statistical language.

Rattle brings together a multitude of $R$ packages that are essential for the data miner but often not easy for the novice to use. An understanding of $R$ is not required in order to get started with Rattlethis will gradually grow as we add sophistication to our data mining. Rattle's user interface provides an entree into the power of $R$ as a data mining tool.

Rattle is used for teaching data mining at numerous universities and is in daily use by consultants and data mining teams world wide. It is also available as a product within Information Builders' WebFocus business intelligence suite as RStat.
Rattle is one of several open source data mining tools (Chen et al., 2007). Many of these tools are also directly available within R (and hence Rattle) through packages like RWeka (Hornik et al., 2009) and arules (Hahsler et al., 2005).

\section{Implementation}

Rattle uses the Gnome graphical user interface as provided through the RGtk2 package (Lawrence and Lang, 2006). It runs under various operating systems, including GNU/Linux, Macintosh OS/X, and MS/Windows.

The GUI itself has been developed using the Glade interactive interface builder. This produces a programming-language-independent XML description of the layout of the widgets that make up the user interface. The XML file is then simply loaded by an application and the GUI is rendered!

Glade allows the developer to freely choose to implement the functionality (i.e., the widget callbacks) in a programming language of choice, and for Rattle that is R. It is interesting to note that the first implementation of Rattle actually used Python for implementing the callbacks and $\mathrm{R}$ for the statistics, using rpy. The release of RGtk2 allowed the interface elements of Rattle to be written directly in $\mathrm{R}$ so that Rattle is a fully R-based application.

Underneath, Rattle relies upon an extensive collection of $\mathrm{R}$ packages. This is a testament to the power of $\mathrm{R}$-it delivers a breadth and depth of statistical analysis that is hard to find anywhere else. Some of the packages underlying Rattle include ada, arules, doBy, ellipse, fBasics, fpc, gplots, Hmisc, kernlab, mice, network, party, playwith, pmml, randomForest, reshape, rggobi, RGtk2, ROCR, RODBC, and rpart. These packages are all available from the Comprehensive $\mathrm{R}$ Archive Network (CRAN). If a package is not installed but we ask through Rattle for some functionality provided by that package, Rattle will popup a message indicating that the package needs to be installed.

Rattle is not only an interface though. Additional functionality that is desired by a data miner has been written for use in Rattle, and is available from the rattle package without using the Rattle GUI. The pmml package (Guazzelli et al., 2009) is an offshoot of the development of Rattle and supports the export of models from Rattle using the open standard XML based PMML, or Predictive Model Markup Language (Data Mining Group, 2008). Models exported from $\mathrm{R}$ in this way can be imported into tools like the ADAPA decision engine running on cloud computers, Teradata's Warehouse Miner for deployment as SQL over a very large database, and Information Builder's WebFocus which handles 
data sourcing, preparation, and reporting, and is able to transform Rattle generated PMML models into C code to run on over 30 platforms.

\section{Installation}

The Gnome and Glade libraries need to be installed (separately to R) to run Rattle. On GNU/Linux and Mac/OSX this is usually a simple package installation. Specifically, for Debian or Ubuntu we install packages like gnome and glade-3. For MS/Windows the self-installing libraries can be obtained from http://downloads.sourceforge.net/ gladewin32. Full instructions are available from http: / / rattle.togaware.com.

After installing the required libraries be sure to restart the $\mathrm{R}$ console to ensure $\mathrm{R}$ can find the new libraries.

Assuming $\mathrm{R}$ is installed we can then install the RGtk2 and rattle packages with:

> install.packages ("RGtk2")

> install.packages ("rattle")

Once installed we simply start Rattle by loading the rattle package and then evaluating the rattle() function:

\section{$>$ library (rattle)}

Rattle: Graphical interface for data mining in $\mathrm{R}$. Version 2.5.0 Copyright (C) 2006-2009 Togaware. Type 'rattle()' to shake, rattle, \& roll your data.

$>$ rattle()

Rattle will pop up a window similar to that in Figure 1 .

The latest development version of Rattle is always available from Togaware:
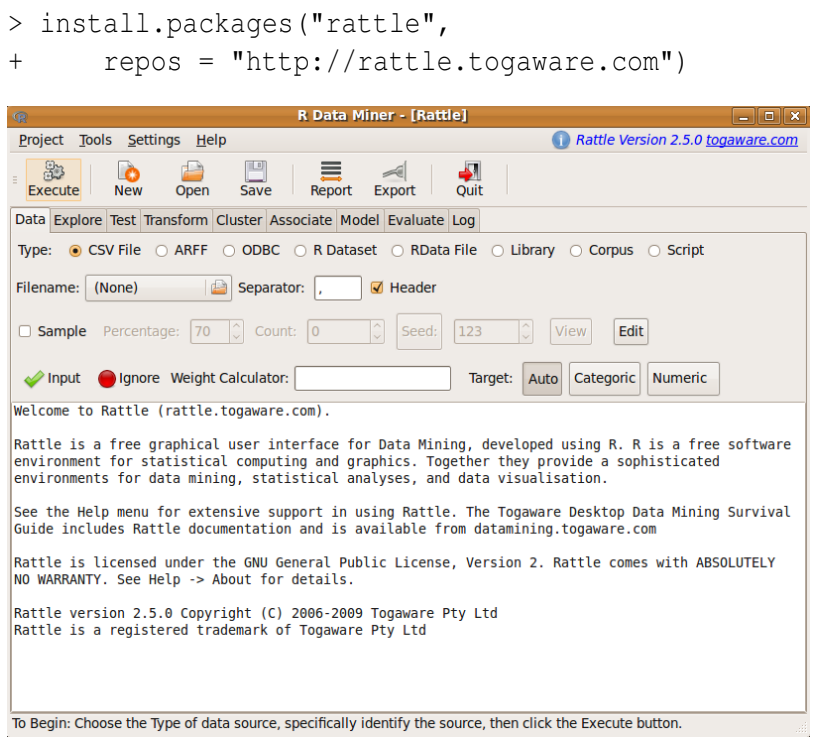

Figure 1: Rattle's introductory screen.

\section{Data Mining}

Rattle specifically uses a simple tab-based concept for the user interface (Figure 1), capturing a work flow through the data mining process with a tab for each stage. A typical work flow progresses from the left most tab (the Data tab) to the right most tab (the Log tab). For any tab the idea is for the user to configure the available options and then to click the Execute button (or F2) to perform the appropriate task. The status bar at the base of the window will indicate when the action is complete.

We can illustrate a very simple, if unrealistic, run through Rattle to build a data mining model with just four mouse clicks. Start up R, load the rattle package, and issue the rattle () command. Then:

1. Click on the Execute button;

2. Click on Yes within the resulting popup;

3. Click on the Model tab;

4. Click on the Execute button.

Now we have a decision tree built from a sample classification dataset.

With only one or two more clicks, alternative models can be built. A few more clicks will have an evaluation chart displayed to compare the performance of the models constructed. Then a click or two more will have the models applied to score a new dataset.

Of course, there is much more to modelling and data mining than simply building a tree model. This simple example provides a flavour of the interface provided by Rattle.

The common work flow for a data mining project can be summarised as:

1. Load a Dataset and select variables;

2. Explore the data to understand distributions;

3. Test distributions;

4. Transform the data to suit the modelling;

5. Build Models;

6. Evaluate models and score datasets;

7. Review the Log of the data mining process.

The underlying R code, constructed and executed by Rattle, is recorded in the Log tab, together with instructive comments. This allows the user to review the actual $\mathrm{R}$ commands. The $\mathrm{R}$ code snippets can also be copied as text (or Exported to file) from the Log tab and pasted into the R console and executed. This allows Rattle to be deployed for basic tasks, yet still access the full power of $R$ as needed (e.g., to fine-tune modelling options that are not exposed in the interface).

The use of Sweave (Leisch, 2002) to allow LATEX markup as the format of the contents of the log is 
experimental but will introduce the concept of literate data mining. The data miner will document their activity, as they proceed through Rattle, by editing the log which is also automatically populated as the modelling proceeds. Simple and automatic processing can then turn the log into a formatted report that also embodies the actual code, which may also be run so as to replicate the activity.

Using the related Tangle processor allows the log to be exported as an R script file, to record the actions taken. The script can then be independently run at a later time (or pasted into the $\mathrm{R}$ console).

Repeatability and reproducibility are important in both scientific research and commercial practice.

\section{Data}

If no dataset has been supplied to Rattle and we click the Execute button (e.g., startup Rattle and immediately click Execute) we are given the option to load one of Rattle's sample datasets from a CSV file.

Rattle can load data from various sources. It directly supports CSV (comma separated data), TXT (tab separated data), ARFF (a common data mining dataset format (Witten and Frank, 2005) which adds type information to a CSV file), and ODBC connections (allowing connection to many data sources including MySQL, SQLite, Postgress, MS/Excel, MS/Access, SQL Server, Oracle, IBM DB2, Netezza, and Teradata). $\mathrm{R}$ data frames attached to the current $R$ session, and datasets available from the packages installed in the $\mathrm{R}$ libraries, are also available through the Rattle interface.

To explore the use of Rattle as a data mining tool we consider the sample audit dataset provided by the rattle package. The data is artificial, but reflects a real world dataset used for reviewing the outcomes of historic financial audits. Picture, for example, a revenue authority collecting taxes based on information supplied by the tax payer. Thousands of random audits might be performed and the outcomes indicate whether an adjustment to the supplied information was required, resulting in a change to the taxpayer's liability.

The audit dataset is supplied as both an R dataset and as a CSV file. The dataset consists of 2,000 fictional tax payers who have been audited for tax compliance. For each case an outcome after the audit is recorded (whether the financial claims had to be adjusted or not). The actual dollar amount of adjustment that resulted is also recorded (noting that adjustments can go in either direction).

The audit dataset contains 13 variables (or columns), with the first being a unique client identifier.

When loading data into Rattle certain special prefixes to variable names can be used to identify default variable roles. For example, if the variable name starts with 'ID_' then the variable is marked as having a role as an identifier. Other prefixes include 'IGNORE_', 'RISK_,' 'IMP_' (for imputed) and 'TARGET_. Examples from the audit data include IGNORE_ACCOunts and TARGET_Adjusted.

The CSV option of the Data tab provides the simplest approach to loading data into Rattle. If the Data tab is Executed with no CSV file name specified then Rattle offers the option to load a sample dataset. Clicking on the Filename box will then list other available sample datasets, including 'audit.csv'.

Once Rattle loads a dataset the text window will contain the list of available variables and their default roles (as in Figure 2).

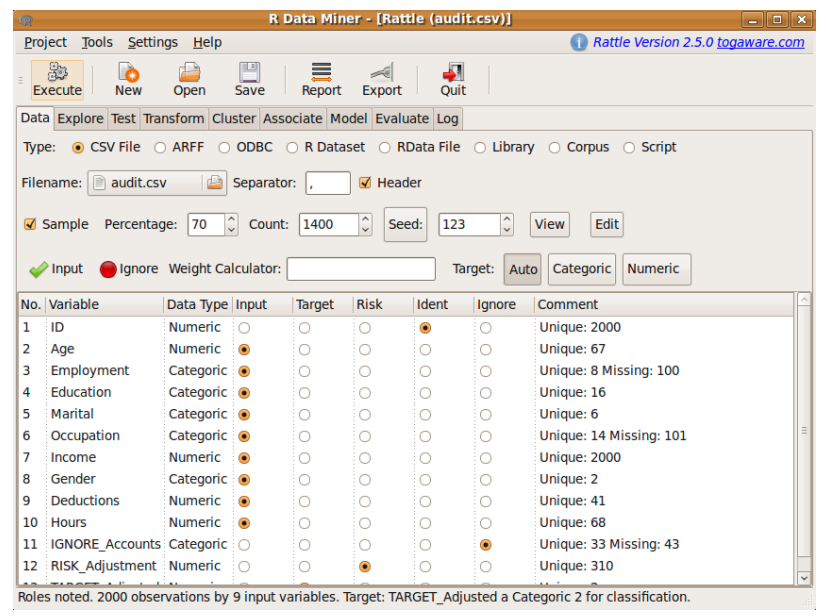

Figure 2: Rattle's variable roles screen.

By default, most variables have a role of Input for modelling. We may want to identify one variable as the Target variable, and optionally identify another variable as a Risk variable (which is a measure of the size of the "targets"). Other roles include Ident and Ignore.

Rattle uses simple heuristics to guess at roles, particularly for the target and ignored variables. For, example, any numeric variable that has a unique value for each observation is automatically identified as an identifier.

Rattle will, by default, partition the dataset into a training and a test dataset. This kind of sampling is useful for exploratory purposes when the data is quite large. Its primary purpose, though, is to select a $70 \%$ sample for training of models, providing a $30 \%$ set for testing.

\section{Explore}

Exploratory data analysis is important in understanding our data. The Explore tab provides numerous numeric and graphic tools for exploring data. Once again, there is a considerable reliance on many other R packages. 


\section{Summary}

The Summary option uses R's summary command to provide a basic univariate summary. This is augmented with the contents and describe commands from the Hmisc package (Harrell, 2009). Extended summaries include additional statistics provided by the fBasics package (Wuertz, 2009), kurtosis and skewness, as well as a summary of missing values using the missing value functionality from the mice package (van Buuren and GroothuisOudshoorn, 2009).

\section{Distributions}

The Distributions option provides access to numerous plot types. It is always a good idea to review the distributions of the values of each of the variables before we consider data mining. While the above summaries help, the visual explorations can often be quite revealing (Cook and Swayne, 2007).

A vast array of tools are available within $\mathrm{R}$ for presenting data visually and the topic is covered in detail in many books including Cleveland (1993). Rattle provides a simple interface to the underlying functionality in $\mathrm{R}$ for drawing some common plots. The current implementation primarily relies on the base graphics provided by $\mathrm{R}$, but may migrate to the more sophisticated lattice (Sarkar, 2008) or ggplot2 (Wickham, 2009).

Some of the canned plots are illustrated in Figure 3. Clockwise we can see a box plot, a histogram, a mosaic plot, and a Benford's Law plot. Having identified a target variable (in the Data tab) the plots include the distributions for each subset of observations associated with each value of the target variable, wherever this makes sense to do so.

\section{GGobi and Latticist}

Rattle provides access to two sophisticated tools for interactive graphical data analysis: GGobi and Latticist.

The GGobi (Cook and Swayne, 2007) visualisation tool is accessed through the rggobi package (Wickham et al., 2008). GGobi will need to be installed on the system separately, and runs under GNU/Linux, OS/X, and MS/Windows. It is available for download from http://www. ggobi.org/.

Ggobi is useful for exploring high-dimensional data through highly dynamic and interactive graphics, especially with tours, scatterplots, barcharts and parallel coordinate plots. The plots are interactive and linked with brushing and identification. The available functionality is extensive, and supports panning, zooming and rotations.

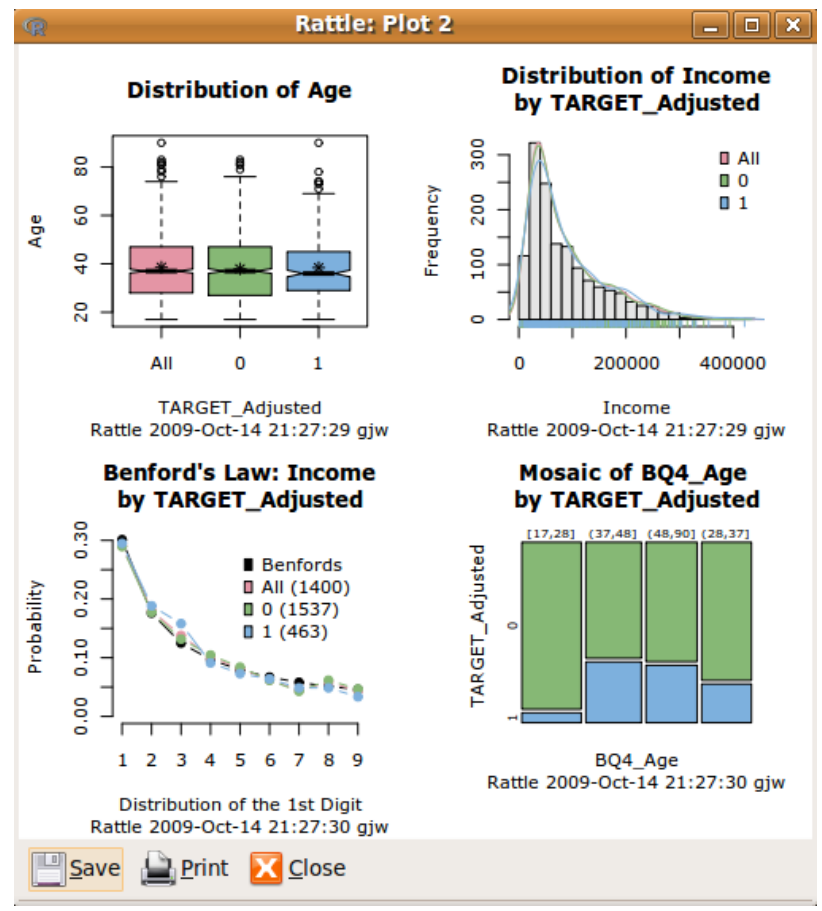

Figure 3: Exploring variable distributions.

Figure 4 displays a scatterplot of Age versus Income (left) and a scatterplot matrix across four variables at the one time (right). Brushing is used to distinguish the class of each observation.
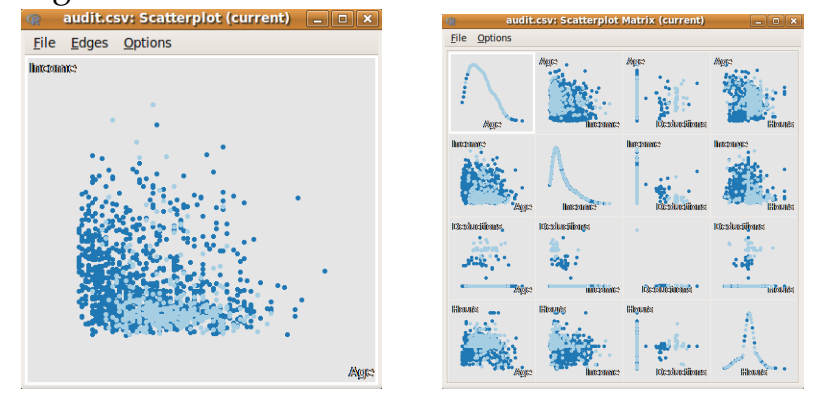

Figure 4: Example of GGobi using rggobi to connect.

A more recent addition to the $\mathrm{R}$ suite of packages are the latticist and playwith packages (Andrews, 2008) which employ lattice graphics within a graphical interface to interactively explore data. The tool supports various plots, data selection and subsetting, and support for brushing and annotations. Figure 5 illustrates the default display when initiated from Rattle.

\section{Test}

The Test tab provides access to a number of parametric and non-parametric statistical tests of distributions. This more recent addition to Rattle continues to receive attention (and hence will change over time). In the context of data mining often applied to the binary classification problem, the current tests are primarily two sample statistical tests. 


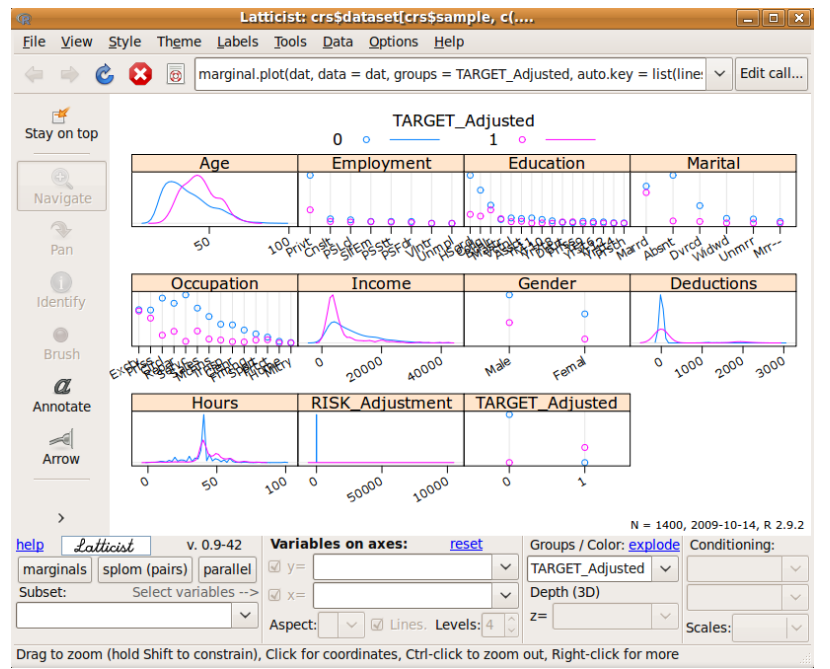

Figure 5: Latticist displaying the audit data.

Tests of data distribution include the Kolomogorov-Smirnov and Wilcoxon Signed Rank tests. For testing the location of the average the Ttest and Wilcoxon Rank-Sum tests are provided. The F-test and Pearson's correlation are also available.

\section{Transform}

Cleaning data and creating new features (derived variables) occupies much time for a data miner. There are many approaches to data cleaning, and a programming language like $\mathrm{R}$ supports them all. Rattle's Transform tab (Figure 6) provides a number of the common options for transforming, including rescaling, skewness reduction, imputing missing values, turning numeric variables into categorical variables, and vice versa, dealing with outliers, and removing variables or observations with missing values. We review a number of the transforms here.

\section{Rescale}

The Rescale option offers a number of rescaling operations, using the scale command from base and the rescaler command from the reshape package (Wickham, 2007). Rescalings include recentering and scaling around zero (Recenter), scaling to 0-1 (Scale $[\mathbf{0 , 1}]$ ), converting to a rank ordering (Rank), robust rescaling around zero using the median (Median/MAD), and rescaling based on groups in the data.

For any transformation the original variable is not modified. A new variable is created with a prefix added to the variable's name to indicate the transformation. The prefixes include 'RRC_, ' $R 01_{-}$', 'RRK_', 'RMD_', and 'RBG_', respectively.

The effect of the rescaling can be examined using the Explore tab (Figure 7). Notice that Rattle overlays bar charts with a density plot, by default.

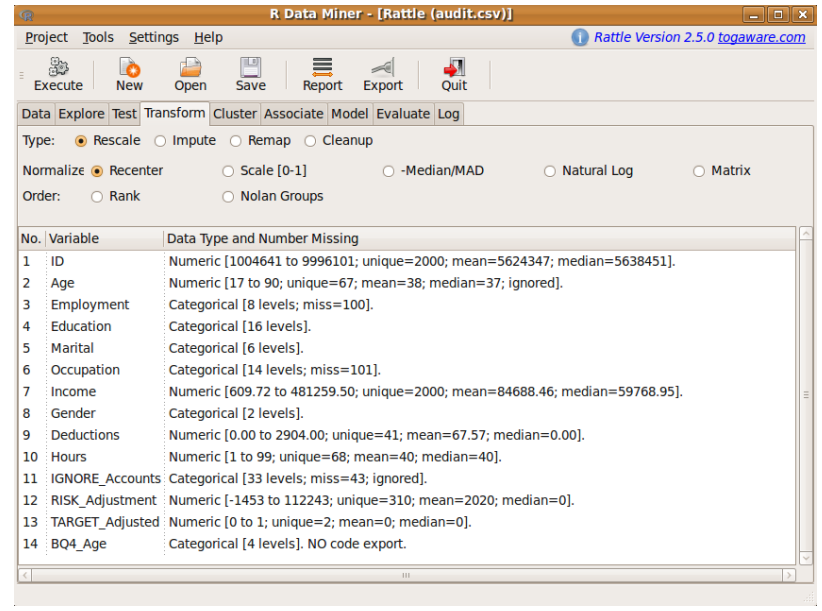

Figure 6: Transform options.

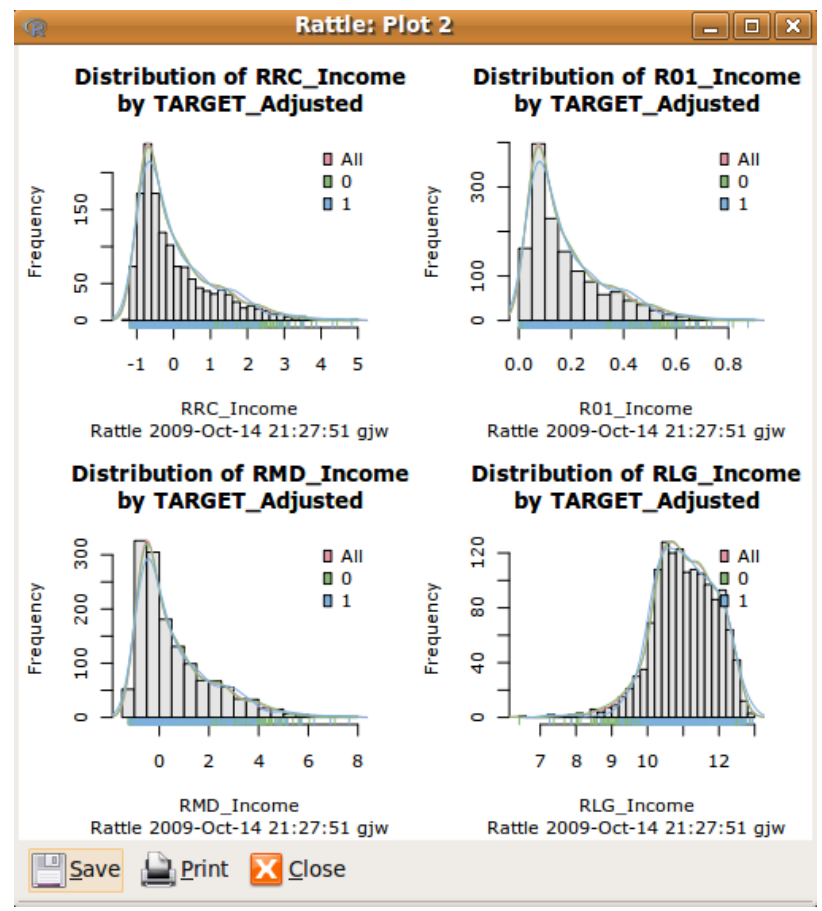

Figure 7: Four rescaled versions of Income.

\section{Impute}

Imputation of missing values is a tricky topic and should only be done with a good understanding of the data. Often, observational data (as distinct from experimental data) will contain missing values, and this can cause a problem for data mining algorithms. For example, the Forest option (using randomForest) silently removes any observation with any missing value! For datasets with a very large number of variables, and a reasonable number of missing values, this may well result in a small, unrepresentative dataset, or even no data at all!

There are many types of imputations possible, only some of which are directly available in Rattle. Further, Rattle does not (yet) support multiple imputation. The pattern of missing values can be viewed 
using the Show Missing check button of the Summary option of the Explore tab.

The simplest, and least recommended, of imputations involves replacing all missing values for a variable with a single value. This makes most sense when we know that the missing values actually indicate that the value is " 0 " rather than unknown. For example, in a taxation context, if a tax payer does not provide a value for a specific type of deduction, then we might assume that they intend it to be zero. Similarly, if the number of children in a family is not recorded, it could be a reasonable assumption that it is zero (but it might equally well mean that the number is just unknown).

A common, if generally unsatisfactory, choice for missing values that are known not to be zero is to use some "central" value of the variable. This is often the mean, median, or mode. We might choose to use the mean, for example, if the variable is otherwise normally distributed (and in particular has little skewness). If the data does exhibit some skewness though (e.g., there are a small number of very large values) then the median might be a better choice.

Be wary of any imputation performed. It is, after all, inventing new data! Future development of Rattle may provide more support with model based imputation through packages like Amelia (Honaker et al., 2009).

\section{Remap}

The Remap option provides numerous re-mapping operations, including binning, log transforms, ratios, and mapping categorical variables into indicator variables for the situation where a model builder requires numeric data. Rattle provides options to use Quantile binning, KMeans binning, and Equal Width binning. For each option the default number of bins is 4 but we can change this to suit our needs. The generated variables are prefixed with either 'BQn_', 'BKn_', and 'BEn_' respectively, with ' $n$ ' replaced with the number of bins. Thus, we can create multiple binnings for any variable.

There are also options to Join Categorics-a convenient way to stratify the dataset, based on multiple categoric variables. A Log transform is also available.

\section{Model}

Data mining algorithms are often described as being either descriptive or predictive. Rattle currently supports the two common descriptive or unsupervised approaches to model building: cluster analysis and association analysis. A variety of predictive model builders are supported: decision trees, boosting, random forests, support vector machines, generalised linear models, and neural networks.
Predictive modelling, and generally the task of classification, is at the heart of data mining. Rattle originally focused on the common data mining task of binary (or two class) classification but now supports multinomial classification and regression, as well as descriptive models.

Rattle provides a straight-forward interface to a collection of descriptive and predictive model builders available in R. For each, a simple collection of tuning parameters is exposed through the graphical interface. Where possible, Rattle attempts to present good default values (often the same defaults as selected by the author of the respective package) to allow the user to simply build a model with no or little tuning. This may not always be the right approach, but is certainly a reasonable place to start.

We will review modelling within Rattle through decision trees and random forests.

\section{Decision Trees}

One of the classic machine learning techniques, widely deployed in data mining, is decision tree induction Quinlan (1986). Using a simple algorithm and a simple tree structure to represent the model, the approach has proven to be very effective. Underneath, the rpart (Therneau et al., 2009) and party (Hothorn et al., 2006) packages are called upon to do the work. Figure 8 shows the Model tab with the results of building a decision tree displayed textually (the usual output from the summary command for an "rpart" object).

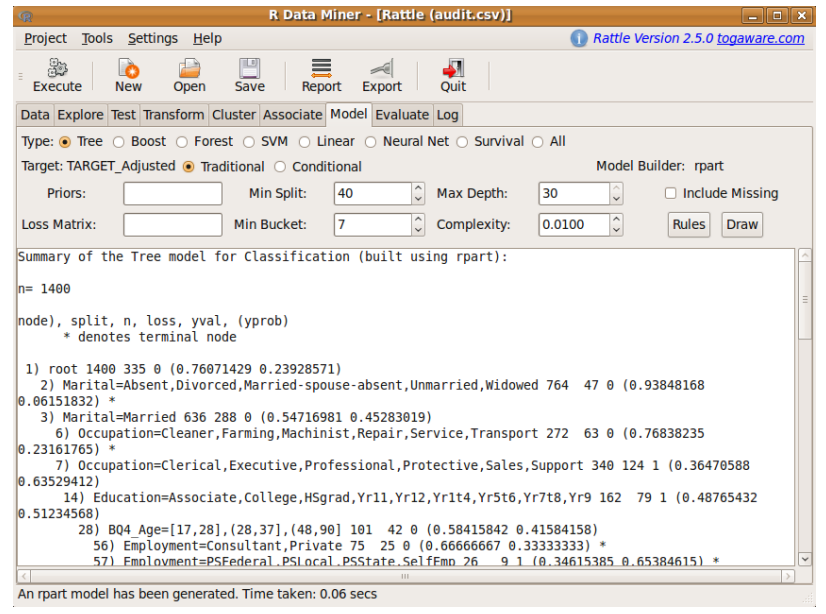

Figure 8: Building a decision tree.

Rattle adds value to the basic rpart functionality with additional displays of the decision tree, as in Figure 9, and the conversion of the decision tree into a list of rules (using the Draw and Rules buttons respectively). 


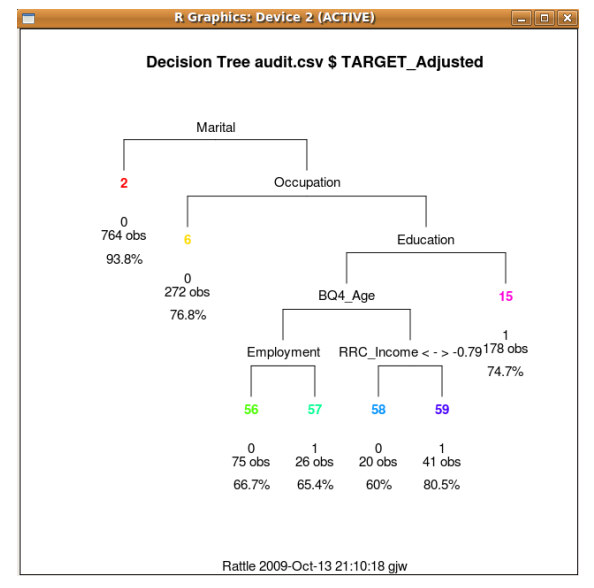

Figure 9: Rattle's display of a decision tree.

\section{Ensemble}

The ensemble approach has gained a lot of interest lately. Early work (Williams, 1988) experimented with the idea of combining a collection of decision trees. The results there indicated the benefit of building multiple trees and combining them into a single model, as an ensemble.

Recent developments continue to demonstrate the effectiveness of ensembles in data mining through the use of the boosting and random forest algorithms. Both are supported in rattle and we consider just the random forest here.

\section{Random Forests}

A random forest (Breiman, 2001) develops an ensemble of decision trees. Random forests are often used when we have very large training datasets and a very large number of input variables (hundreds or even thousands of input variables). A random forest model is typically made up of tens or hundreds of decision trees, each built using a random sample of the dataset, and whilst building any one tree, a random sample of the variables is considered at each node.

The random sampling of both the data and the variables ensures that even building 500 decision trees can be efficient. It also reputably delivers considerable robustness to noise, outliers, and overfitting, when compared to a single tree classifier.

Rattle uses the randomForest package (Liaw and Weiner, 2002) to build a forest of trees. This is an interface to the original random forest code from the original developers of the technique. Consequently though, the resulting trees have a different structure to standard "rpart" trees, and so some of the same tree visualisations are not readily available. Rattle can list all of the rules generated for a random forest, if required. For complex problems this can be a very long list indeed (thousands of rules).

The Forest option can also display a plot of relative variable importance. This provides insight into which variables play the most important role in predicting the class outputs. The Importance button will display two plots showing alternative measures of the relative importance of the variables in our dataset in relation to predicting the class.

\section{Building All Models and Tuning}

Empirically, the different model builders often produce models that perform similarly, in terms of misclassification rates. Thus, it is quite instructive to use all of the model builders over the same dataset. The All option will build one model for each of the different model builders.

We can review the performance of each of the models built and choose that which best suits our needs. In choosing a single model we may not necessarily choose the most accurate model. Other factors can come into play. For example, if the simple decision tree is almost as accurate as the 500 trees in the random forest ensemble, then we may not want to step up to the complexity of the random forest for deployment.

An effective alternative, where explanations are not required, and accuracy is desired, is to build a model of each type and to then build an ensemble that is a linear combination of these models.

\section{Evaluate}

Rattle provides a standard collection of tools for evaluating and comparing the performance of models. This includes the error matrix (or confusion table), lift charts, ROC curves, and Cost Curves, using, for example, the ROCR package (Sing et al., 2009). Figure 10 shows the options.

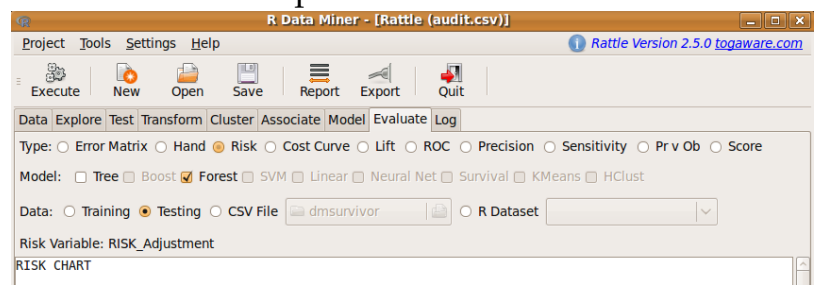

Figure 10: Options for Evaluation.

A cumulative variation of the ROC curve is implemented in Rattle as Risk charts (Figure 11). Risk charts are particularly suited to binary classification tasks, which are common in data mining. The aim is to efficiently display an easily understood measure of the performance of the model with respect to resources available. Such charts have been found to be more readily explainable to decision-making executives.

A risk chart is particularly useful in the context of the audit dataset, and for risk analysis tasks in general. The audit dataset has a two class target variable 
as well as a so-called risk variable, which is a measure of the size of the risk associated with each observation. Observations that have no adjustment following an audit (i.e., clients who have supplied the correct information) will of course have a risk of zero associated with them. Observations that do have an adjustment will usually have a risk associated with them, and for convenience we simply identify the value of the adjustment as the magnitude of the risk.

Rattle uses the idea of a risk chart to evaluate the performance of a model in the context of risk analysis.

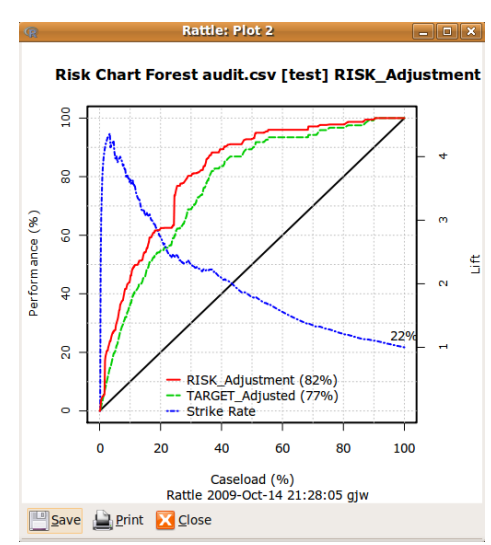

Figure 11: A simple cumulative risk chart.

A risk chart plots performance against caseload. Suppose we had a population of just 100 observations (or audit cases). The case load is the percentage of these cases that we will actually ask our auditors to process. The remaining cases will not be considered any further, expecting them to be low risk, and hence, with limited resources, not requiring any action.

The decision as to what percentage of cases are actioned corresponds to the $\mathrm{x}$-axis of the risk chartthe caseload. A 100\% caseload indicates that we will action all audit cases. A $25 \%$ caseload indicates that we will action just one quarter of all cases.

The performance is the percentage of the total number of cases that required an adjustment (or the total risk-both are plotted if a risk variable is identified) that might be covered in the population that we action.

The risk chart allows the trade-off between resources and risk to be visualised.

\section{Model Deployment}

Once we have decided upon a model that represents acceptable improvement to our business processes we are faced with deployment. Deployment can range from running the model ad hoc, to a fully automated and carefully governed deployment environment. We discuss some of the issues here and explore how Rattle supports deployment.

\section{Scripting R}

The simplest approach to deployment is to apply the model to a new dataset. This is often referred to as scoring. In the context of $\mathrm{R}$ this is nothing more than using the predict function.

Rattle's evaluation tab supports scoring with the Score option. There are further options to score the training dataset, the test dataset, or data loaded from a CSV file (which must contain the exact same variables). Any number of models can be selected, and the results are written to a CSV file.

Scoring is often performed some time after the model is built. In this case the model needs to be saved for later use. The concept of a Rattle project is useful in such a circumstance. The current state of Rattle (including the actual data and models built during a session) can be saved to a project, and later loaded into a new instance of Rattle (running on the same host or even a different host and operating system). A new dataset can then be scored using the saved model.

Underneath, saving/loading a Rattle project requires no more than using the save and load commands of $\mathrm{R}$ to create a binary representation of the $\mathrm{R}$ objects, and saving them to file. A Rattle project can get quite large, particularly with large datasets.

Larger files take longer to load, and for deploying a model it is often not necessary to keep the original data. So as we get serious about deployment we might save just the model we wish to deploy. This is done using the save function and knowing a little bit about the internals of Rattle (but no more than what is exposed through the Log tab).

The approach, saving a randomForest model, might be:

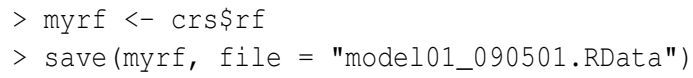

We can then load the model at a later time and apply the model (using a script based on the commands shown in the Rattle Log tab) to a new dataset:

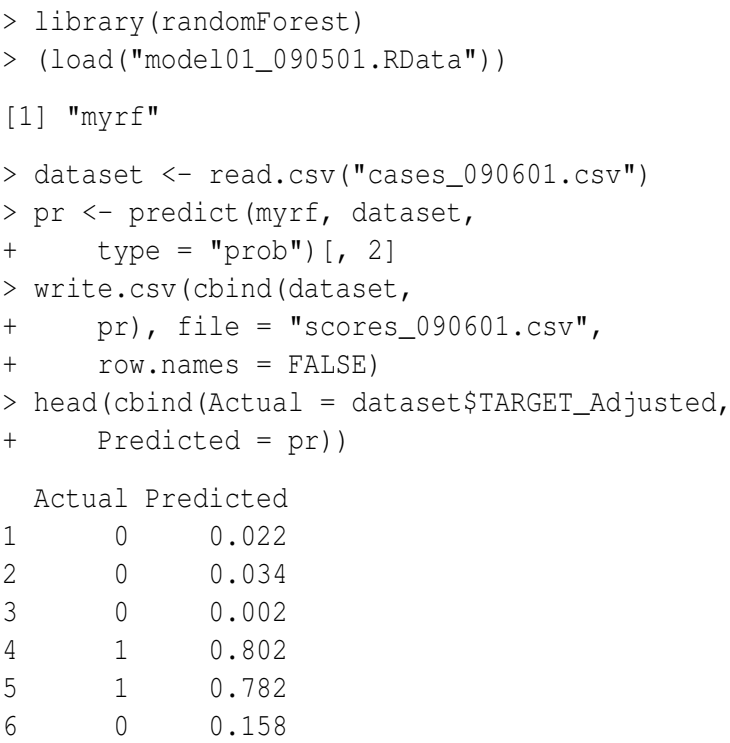


As an aside, we can see the random forest model is doing okay on these few observations.

In practise (e.g., in the Australian Taxation Office) once model deployment has been approved the model is deployed into a secure environment. It is scheduled regularly to be applied to new data using a script that is very similar to that above (using the littler package for GNU/Linux). The data is obtained from a data warehouse and the results populate a data warehouse table which is then used to automatically generate work items for auditors to action.

\section{Export to PMML}

An alternative approach to deployment is to export the model so that it can be imported into other software for prediction on new data.

We have experimented with exporting random forests to $\mathrm{C}++$ code. This has been demonstrated running over millions of rows of new data in a data warehouse in seconds.

Exporting to a variety of different languages, such as $\mathrm{C}++$, is not an efficient approach to exporting models. Instead, exporting to a standard representation, which other software can also export, makes more sense. This standard representation can then be exported to a variety of other languages.

The Predictive Model Markup Language (Data Mining Group, 2008) provides such a standard language for representing data mining models. PMML is an XML based standard that is supported, to some extent, by the major commercial data mining vendors and many open source data mining tools.

The pmml package for $\mathrm{R}$ was separated from the rattle package to allow its independent development with contributions from a broader community. PMML models generated by Rattle, using the pmml package, can be imported into a number of other products, including Teradata Warehouse Miner (which converts models to SQL for execution), Information Builders' WebFocus (which converts models to $C$ code for execution on over 30 platforms), and Zementis' ADAPA tool for online execution.

The Export button (whilst displaying a model within the Model tab) will export a model as PMML.

\section{$\log$}

A GUI is not as complete and flexible as a full programming language. Rattle is sufficient for many data miners, providing a basic point-and-click environment for quick and consistent data mining, gaining much from the breadth and depth of R. However, a professional data miner will soon find the need to go beyond the assumptions embodied in Rattle. Rattle supports this through the Log tab.
As mentioned above, a log of the $\mathrm{R}$ commands that Rattle constructs are exposed through the Log tab. The intention is that the $\mathrm{R}$ commands be available for copying into the $\mathrm{R}$ console so that where Rattle only exposes a limited number of options, further options can be tuned via the $\mathrm{R}$ console.

The Log tab captures the commands for later execution and is also educational. Informative comments are included to describe the steps involved. The intention is that it provide a tutorial introduction to using $\mathrm{R}$ from the command line, where we obtain a lot more power.

The text that appears at the top of the Log tab is shown in Figure 12. Commentary text is preceded with R's comment character (the \#), with R commands in between.

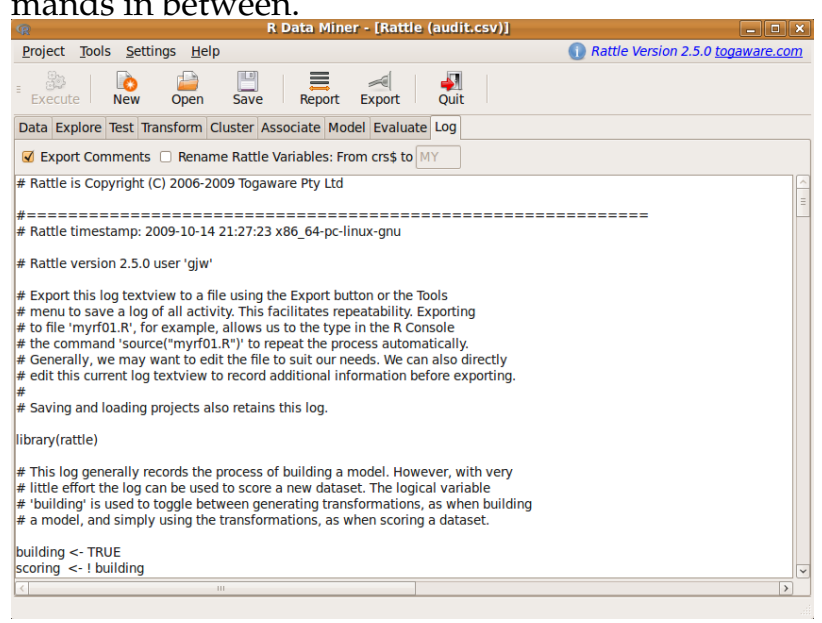

Figure 12: Rattle Log.

The whole log can be exported to a script file (with a '. $R$ ' filename extension) and then loaded into $\mathrm{R}$ or an $\mathrm{R}$ script editor (like Emacs/ESS or Tinn-R) to repeat the exact steps of the Rattle interactions. In general, we will want to review the code and finetune it to suit our purposes. After exporting the Log tab into a file, with a filename like 'myrf01. $R^{\prime}$, we can have the file execute as a script in $\mathrm{R}$ with:

> source("myrf01.R")

\section{Help}

The Help menu provides access to brief descriptions of the functionality of Rattle, structured to reflect the user interface. Many of the help messages then provide direct access to the underlying documentation for the various packages involved.

\section{Future}

Rattle continues to undergo development, extending in directions dictated by its actual use in data mining projects, and from suggestions and code offered by its user population. Here we mention some of the 
experimental developments that may appear in Rattle over time.

A number of newer $R$ packages provide capabilities that can enhance Rattle significantly. The party package and associated efforts to unify the representation of decision tree models across $\mathrm{R}$ is an exciting development. The caret package offers a unified interface to running a multitude of model builders, and significant support for tuning models over various parameter settings. This latter capability is something that has been experimented with in Rattle, but not yet well developed.

A text mining capability is in the pipeline. Current versions of Rattle can load a corpus of documents, transform them into feature space, and then have available all of Rattle's capabilities. The loading of a corpus and its transformation into feature space relies on the tm package (Feinerer, 2008).

Time series analysis is not directly supported in Rattle. Such a capability will incorporate the ability to analyse web log histories and observations of many entities over time.

Spatial data analysis is another area of considerable interest, often at the pre-processing stage of data mining. The extensive work completed for spatial data analysis with R (Bivand et al., 2008) may provide the basis for extending Rattle in this direction.

Further focus on missing value imputation is likely, with the introduction of more sound approaches, including k-nearest neighbours and multiple imputation.

Initial support for automated report generation using the odfWeave package is included in Rattle (through the Report button). Standard report templates are under development for each of the tabs. For the Data tab, for example, the report provides plots and tables showing distributions and basic statistics.

The Rattle code will remain open source and others are welcome to contribute. The source code is hosted by Google Code (http://code.google.com/ $\mathrm{p} / \mathrm{rattle} /$ ). The Rattle Users mailing list (http: //groups.google.com/group/rattle-users) is also hosted by Google. An open source reference book is also available (Williams, 2009a).

\section{Acknowledgements}

A desire to see $\mathrm{R}$ in the hands of many more of my colleagues at the Australian Taxation Office lead to the development of Rattle. I thank Stuart Hamilton, Frank Lu and Anthony Nolan for their ongoing encouragement and feedback. Michael Lawrence's work on the RGtk2 package provided a familiar platform for the development of GUI applications in R.

The power of Rattle relies on the contributions of the open source community and the underlying $\mathrm{R}$ packages. For the online version of this article, fol- low the package links to find many of those who deserve very much credit.

We are simply standing on the shoulders of those who have gone before us, potentially providing new foundations for those who follow this way.

\section{Bibliography}

F. Andrews. latticist: A graphical user interface for exploratory visualisation, 2008. URL http:// latticist.googlecode.com/. R package version $0.9-42$.

R. S. Bivand, E. J. Pebesma, and V. Gómez-Rubio. Applied Spatial Data Analysis with R. Use R! Springer, New York, 2008. ISBN 978-0-387-78170-9.

L. Breiman. Random forests. Machine Learning, 45(1): 5-32, 2001.

S. van Buuren and K. Groothuis-Oudshoorn. MICE: Multivariate Imputation by Chained Equations in R Journal of Statistical Software, forthcoming, 2009. URL http: //CRAN.R-project.org/package=mice.

X. Chen, G. Williams, and X. Xu. Open source data mining systems. In Z. Huang and Y. Ye, editors, Proceedings of the Industry Stream, 11th Pacific Asia Conference on Knowledge Discovery and Data Mining (PAKDD07), 2007.

W. S. Cleveland. Visualizing Data. Hobart Press, Summit, New Jersey, 1993. ISBN 0-9634884-0-6.

D. Cook and D. F. Swayne. Interactive and Dynamic Graphics for Data Analysis. Springer, 2007.

Data Mining Group. PMML version 3.2. WWW, 2008. URL http: //www. dmg.org/pmml-v3-2.html.

I. Feinerer. An introduction to text mining in R. $R$ News, 8(2):19-22, Oct. 2008.

A. Guazzelli, M. Zeller, W.-C. Lin, and G. Williams. Pmml: An open standard for sharing models. The $R$ Journal, 1(1):60-65, May 2009. URL http://journal.r-project.org/2009-1/ RJournal_2009-1_Guazzellitet+al.pdf.

M. Hahsler, B. Gruen and K. Hornik arules - A computational environment for mining association rules and frequent item sets. Journal of Statistical Software, 14/15, 2005.

F. E. Harrell Jr and with contributions from many other users. Hmisc: Harrell Miscellaneous, 2009 URL http: //CRAN.R-project.org/package=Hmisc. R package version 3.7-0.

J. Honaker, G. King, and M. Blackwell. Amelia: Amelia II: A Program for Missing Data, 2009. URL http://CRAN.R-project.org/package=Amelia. R package version 1.2-13. 
K. Hornik, C. Buchta, and A. Zeileis. Open-Source machine learning: $\mathrm{R}$ meets Weka. Computational Statistics, 24(2):225-232, 2009.

T. Hothorn, K. Hornik and A. Zeileis. Unbiased recursive partitioning: A conditional inference framework. Journal of Computational and Graphical Statistics, 15(3):651-674, 2006.

M. Lawrence and D. T. Lang. Rgtk2-a GUI toolkit for R. Statistical Computing and Graphics, 17(1), 2006.

F. Leisch. Sweave: Dynamic generation of statistical reports using literate data analysis. In W. Härdle and B. Rönz, editors, Compstat 2002 - Proceedings in Computational Statistics, pages 575-580. Physica Verlag, Heidelberg, 2002. URL http: //www . stat. uni-muenchen. de/ leisch/Sweave. ISBN 3-79081517-9.

A. Liaw and M. Wiener. Classification and regression by randomForest. R News, 2(3), 2002.

J. R. Quinlan. Induction of decision trees. Machine Learning, 1(1):81-106, 1986.

D. Sarkar. Lattice: Multivariate Data Visualization with $R$. Use R! Springer, New York, 2008. ISBN 978-0387-75968-5.

T. Sing, O. Sander, N. Beerenwinkel and T. Lengauer. ROCR: Visualizing the performance of scoring classifiers, 2009. URL http://CRAN.R-project.org/ package=ROCR. R package version 1.0-3.

T. M. Therneau and B. Atkinson. R port by B. Ripley. rpart: Recursive Partitioning, 2009. URL http: //CRAN.R-project.org/package=rpart. R package version 3.1-45.
H. Wickham. Reshaping data with the reshape package. Journal of Statistical Software, 21(12), 2007.

H. Wickham. goplot2 Elegant Graphics for Data Analysis. Use R! Springer, New York, 2008. ISBN 978-0387-75968-5.

H. Wickham, M. Lawrence, D. T. Lang, and D. F. Swayne. An introduction to rggobi. R News, 8(2): 3-7, Oct. 2008.

G. J. Williams. The Data Mining Desktop Survival Guide. Togaware, 2009a. URL http: // datamining. togaware.com/survival.

G. J. Williams. rattle: A graphical user interface for data mining in $R, 2009 \mathrm{~b}$. URL http: / / cran.r-project. org/package=rattle. R package version 2.4.55.

G. J. Williams. Combining decision trees: Initial results from the MIL algorithm. In J. S. Gero and R. B. Stanton, editors, Artificial Intelligence Developments and Applications, pages 273-289. Elsevier Science Publishers B.V. (North-Holland), 1988.

I. H. Witten and E. Frank. Data Mining: Practical Machine Learning Tools and Techniques. Morgan Kaufmann, San Francisco, 2nd edition, 2005. URL http: //www. cs. waikato.ac.nz/ ml/weka/book.html.

D. Wuertz and many others. fBasics: Rmetrics - Markets and Basic Statistics, 2009 URL http://CRAN. $\mathrm{R}$-project.org/package=fBasics. R package version 2100.78 .

\section{Graham Williams}

Togaware Pty Ltd

Graham.Williams@togaware.com 